\title{
A modified Larson's method of posterolateral corner reconstruction of the knee reproducing the physiological tensioning pattern of the lateral collateral and popliteofibular ligaments
}

\author{
Yasuo Niki, Hideo Matsumoto, Toshiro Otani, Hiroyuki Enomoto, Yoshiaki Toyama and Yasunori Suda
}

\begin{abstract}
Background: Consensus has been lacking as to how to reconstruct the posterolateral corner (PLC) of the knee in patients with posterolateral instability. We describe a new reconstructive technique for PLC based on Larson's method, which reflects the physiological load-sharing pattern of the lateral collateral ligament $(\mathrm{LCL})$ and popliteofibular ligament (PFL).

Findings: Semitendinosus graft is harvested, and one limb of the graft comprises PFL and the other comprises $L C L$. Femoral bone tunnels for the $L C L$ and popliteus tendon are made at their anatomical insertions. Fibular bone tunnel is prepared from the anatomical insertion of the $L C L$ to the proximal posteromedial portion of the fibular head, which corresponds to the insertion of the PFL. The graft end for popliteus tendon is delivered into the femoral bone tunnel and secured on the medial femoral condyle. The other end for $L C L$ is passed through the fibular tunnel from posterior to anterior. While the knee is held in 90 of flexion, the graft is secured in the fibular tunnel using a $5 \mathrm{~mm}$ interference screw. Then, the $L C L$ end is passed into the femoral bone tunnel and secured at the knee in extension.

Conclusions: Differential tension patterns between $L C L$ and PFL is critical when securing these graft limbs. Intrafibular fixation of the graft using a small interference screw allows us to secure these two graft limbs independently with intended tension at the intended flexion angle of the knee.
\end{abstract}

Keywords: Posterolateral corner, Lateral collateral ligament, Popliteofibular ligament, Reconstructive technique

\section{Introduction}

Generally, posterolateral corner (PLC) reconstruction is performed to treat chronic posterolateral instability in patients with PLC injury. However, consensus has been lacking as to how to reconstruct the PLC. Abundant surgical procedures for PLC have been accumulated, and can be broadly divided into two types: anatomical and non-anatomical. Non-anatomical reconstructions include biceps tenodesis [1,2], arcuate complex [3], proximal bone block advancements [4], and extracapsular iliotibial band sling [5]. However, current techniques have shifted to more anatomical reconstruction of the

\footnotetext{
* Correspondence: y-niki@sc.itc.keio.ac.jp

Department of Orthopaedic Surgery, Keio University, 35 Shinanomachi, Shinjuku-ku, Tokyo 160-8582, Japan
}

three major functional components of the PLC: the lateral collateral ligament (LCL), popliteofibular ligament (PFL), and popliteus tendon [6-9]. We have developed a new reconstructive technique for PLC based on Larson's method [10], which reflects the physiological loadsharing pattern of the LCL and PFL. This technique is less invasive and less technically demanding than current anatomical reconstructive techniques.

\section{Surgical techniques}

The patient is positioned supine on the operating table with an arthroscopic leg holder, after precise diagnosis of posterolateral instability and concomitant injuries. Arthroscopy is performed to identify lateral drivethrough sign as well as concomitant disruption of the 


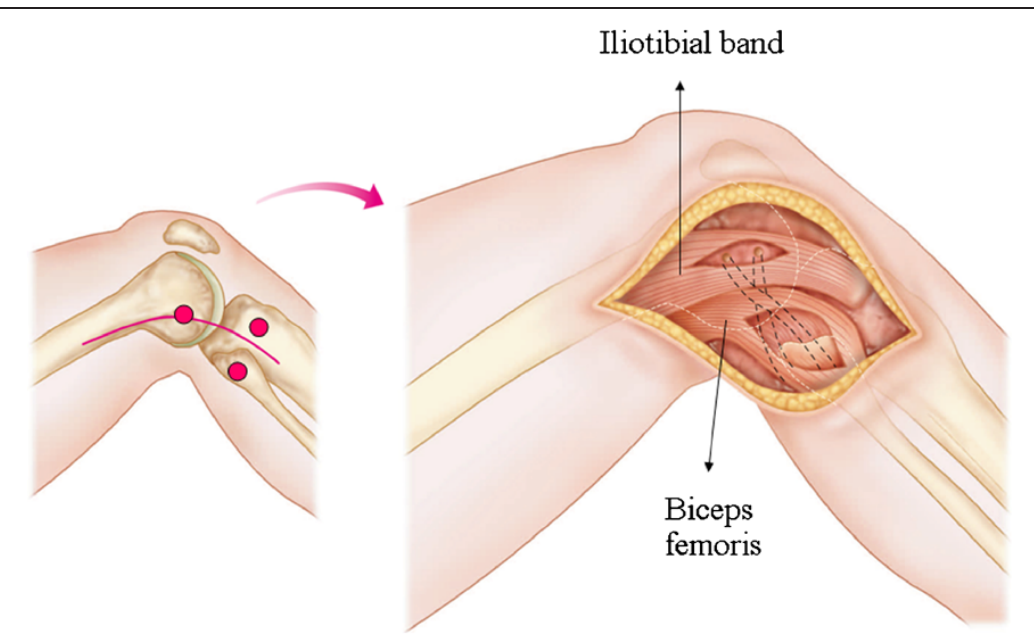

Figure 1 Schematic representation of surgical landmarks over the skin (left panel). Incision is made down to the layer of the iliotibial band and biceps femoris to expose the lateral epicondyle and fibular head, respectively (right panel).

anterior cruciate ligament (ACL) or posterior cruciate ligament (PCL). If the decision has been made to reconstruct either of the cruciate ligaments, this should be performed first. After cruciate ligament reconstruction, PLC reconstruction is initiated. Semitendinosus (ST) tendon is normally harvested ipsilaterally using a smooth tendon stripper, but if ipsilateral ST tendon is planned for use as either PCL or ACL graft, the graft for PLC is harvested from the contralateral ST tendon. The appropriate length of ST graft for PLC is $16-19 \mathrm{~cm}$, which typically reflects the distance between the femoral and fibular insertions of the PLC plus an additional $30 \mathrm{~mm}$, as both ends of the graft require at least $15 \mathrm{~mm}$ each, corresponding to the insertion into bone tunnels. One limb of the graft comprises PFL and the other comprises LCL. A baseball glove suture using FiberWire $^{\text {TM }}$ (Arthrex, Naples, FL) is carried used at the both ends of the graft, and one end of the popliteus tendon is connected to an Endobutton ${ }^{\mathrm{TM}}$ (Smith \& Nephew, Memphis, TN), then placed within antibiotic-soaked gauzes and set aside for later use.

A lateral incision is started just proximal to the lateral femoral epicondyle and followed distally to the midpoint between Gerdy's tubercle and the fibular head (Figure 1). Further incision is performed down to the deep layer in line with fibers of the iliotibial band from the lateral femoral epicondyle to Gerdy's tubercle. After femoral attachments of the LCL and popliteus tendon are exposed, both tendon and ligament are taken off the femur. For exposure of fibular attachments of the LCL and PFL, an incision is made on the biceps femoris muscle in line with this fiber so that the fibular head is well exposed. Anatomical insertions of the LCL and popliteus tendon at the lateral femoral condyle are drilled with 2.4-mm guide pins aimed toward the flare of the medial femoral epicondyle, followed by overdrilling using a reamer matched to the diameter of the graft (typically 4-5 mm) (Figure 2).

The posterolateral aspect of the fibular head is exposed, and attachments of the LCL and PFL are identified. The peroneal nerve should be identified, marked with tape, and retracted with the biceps muscle. To prepare the fibular tunnel, the starting point is set at the distal anterolateral portion of the fibular head, corresponding to the anatomical insertion of the LCL, and the guide pin should exit the proximal posteromedial portion of the fibular head, which corresponds to the

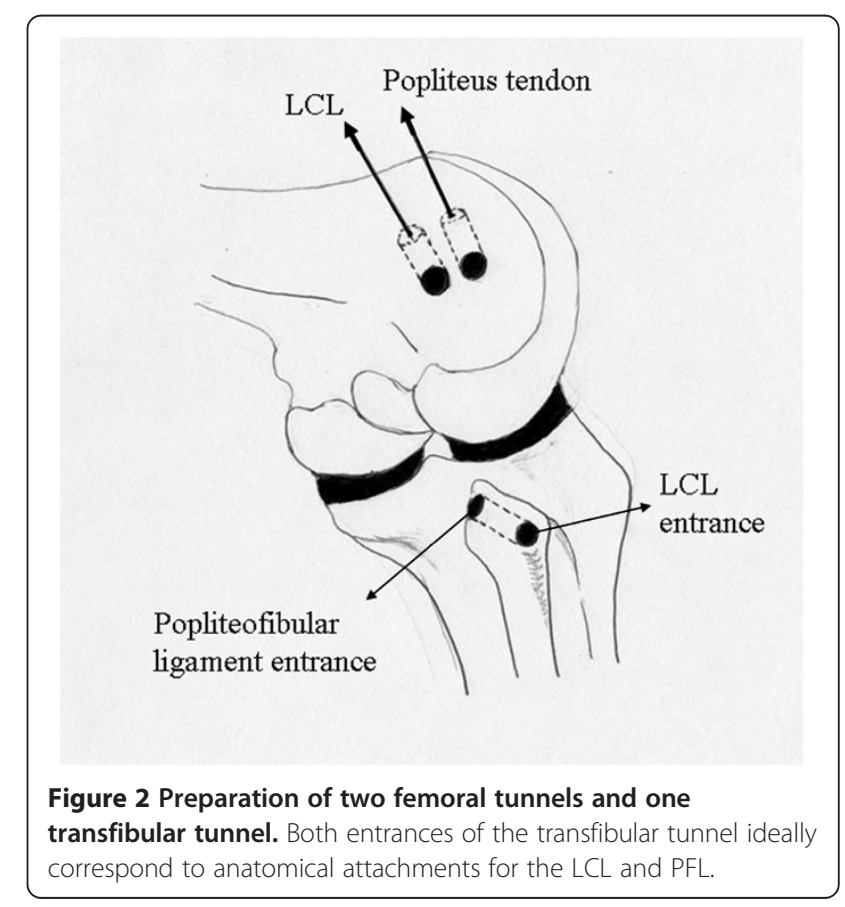


insertion of the PFL. When the patient is of short stature and the fibular head is small, the location of the transfibular tunnel, particularly the LCL insertion site, should be changed from the anatomical site slightly anteriorly to avoid any risk of avulsing the fibular head with the reamer (Figure 3A). At this time, the pattern of length change between the femoral and fibular bone tunnels during knee flexion and extension should be confirmed for both LCL and PFL (Figure 3B). Ideally, the length of LCL will shorten with increasing flexion angle of the knee, while the length of popliteus tendon will increase with increasing flexion angle.

The graft end for PFL connected to the Endobutton ${ }^{\mathrm{TM}}$ is delivered into the femoral bone tunnel and flipped on the cortex of the medial femoral condyle. The other end for LCL is then delivered under the iliotibial band and biceps femoris and is passed through the fibular tunnel from posterior to anterior (Figure 4A). As the LCL graft end is strained manually, the knee is taken through several cycles of full flexion and extension. While the knee is held in $90^{\circ}$ of flexion and the tibia is in a neutral rotation, the graft is then secured in the fibular tunnel with a metal interference screw $5 \mathrm{~mm}$ in diameter (TJ screw; Meira, Nagoya, Japan) with $10 \mathrm{~N}$ of force applied on the graft by use of a ligament tensioner (Smith \& Nephew Endoscopy) (Figure 4B). The interference screw is inserted into the fibular bone tunnel through anteroposterior direction. The graft end for the LCL is then delivered under the biceps and ITB, and is passed into the femoral bone tunnel from the lateral epicondyle to the medial cortex of the femur (Figure 5A). The leading FiberWire $^{\mathrm{TM}}$ sticks out of the medial skin and is tensioned manually. After performing several cycles of full flexion and extension to provide pretension, graft fixation to the bone is accomplished using the $5-\mathrm{mm}$ interference screw (TJ screw) with $10 \mathrm{~N}$ force applied to the graft at the knee in extension and neutral rotation by use of a ligament tensioner (Smith \& Nephew Endoscopy) (Figure 5B). To allow adequate interference screw fixation within tunnels, at least $15 \mathrm{~mm}$ of graft should be positioned within the tunnel. Full flexion and extension are then verified, and improved knee stability is confirmed, particularly for external rotation at knee in $30^{\circ}$ and $90^{\circ}$ of flexion and varus stability at the knee in $0^{\circ}$ and $30^{\circ}$ of flexion. Typically, postoperative radiography shows the Endobutton ${ }^{\mathrm{TM}}$ connected to the graft end of the PFL located in the anterolateral cortex of the femur (Figure 6B). Importantly, in cases following either ACL or PCL reconstruction, particularly double-bundle reconstruction, the bone tunnel for the LCL and PFL should be absolutely prevented from overlapping with either of the two bone tunnels for cruciate ligament.

\section{Postoperative program}

The postoperative program is normally dictated by the cruciate ligament reconstruction, particularly for PCL reconstruction. The affected knee joint is immobilized in a hinged knee brace locked in extension for 2 weeks postoperatively. Range

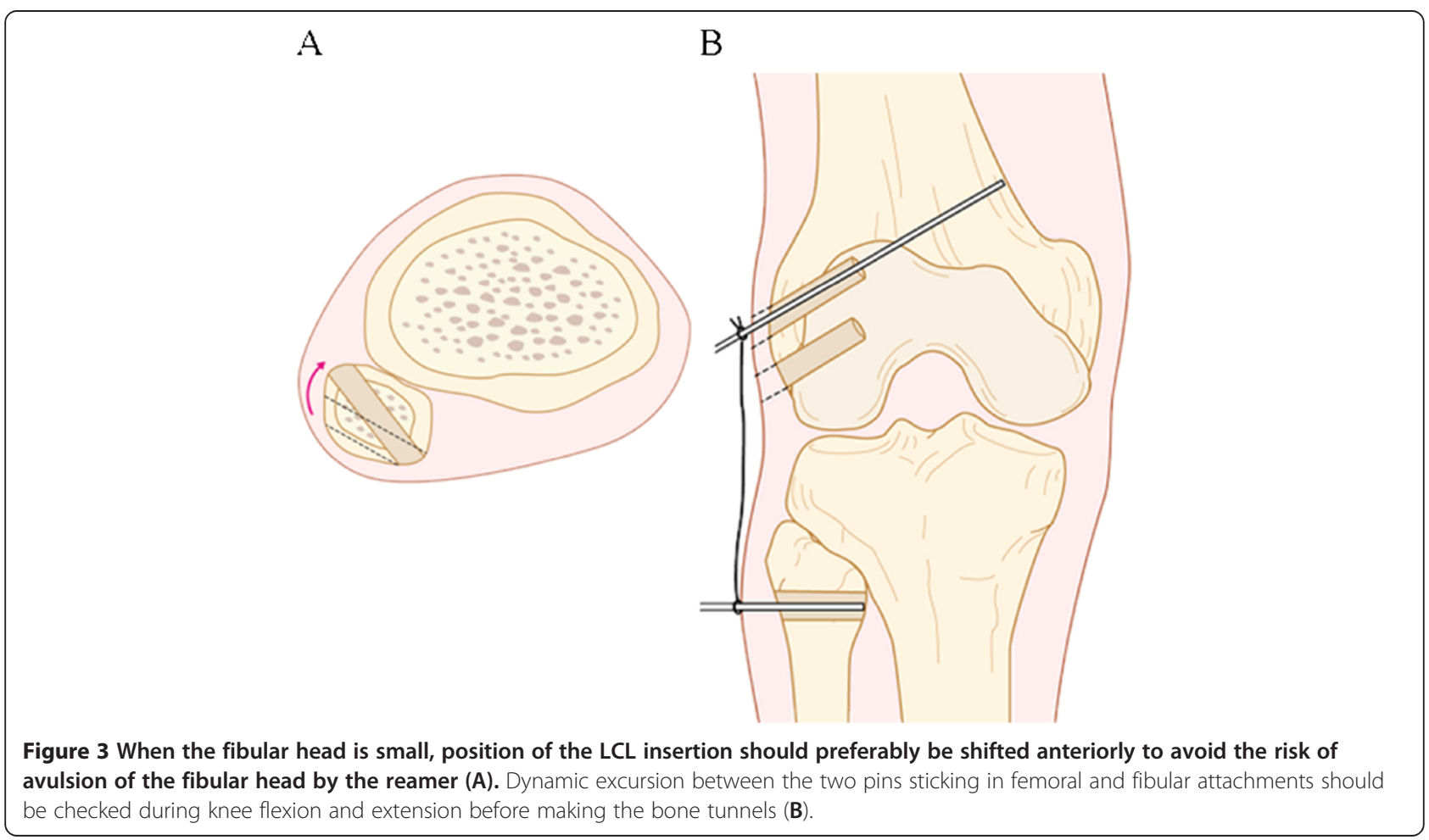


A

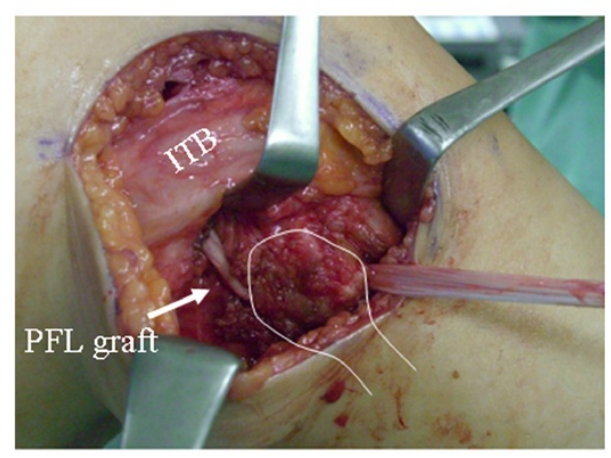

B

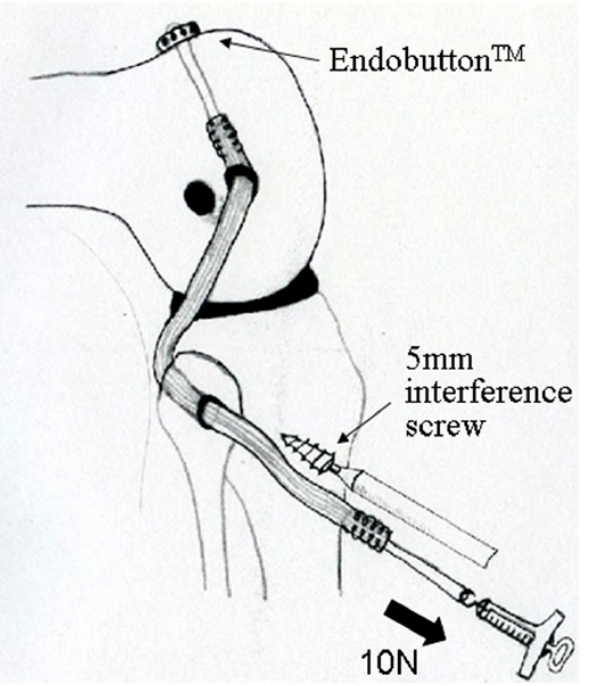

Figure 4 Semitendinosus tendon graft has been secured within the popliteus femoral tunnel using an Endobutton ${ }^{\mathrm{TM}}$, delivered below the ITB, and passed through the transfibular tunnel (A). The graft is fixed in the fibular tunnel with a metal interference screw under $10 \mathrm{~N}$ force of pretension at $90^{\circ}$ knee flexion (B).

of motion (ROM) exercises are initiated using a continuous passive motion (CPM) device and are permitted from $0^{\circ}$ to $90^{\circ}$ of flexion during weeks 3 and 4 . From week 4 on, $>90^{\circ}$ of flexion is permitted. The knee is maintained at $0^{\circ}$ except during ROM exercises. A hinged functional brace is used for 3 months postoperatively. Partial weight-bearing with the brace locked in extension is initiated at 2 weeks postoperatively, with gradual progression to full weight-bearing by 4 weeks postoperatively.

Written informed consent was obtained from the patient for publication of this report and any accompanying images.
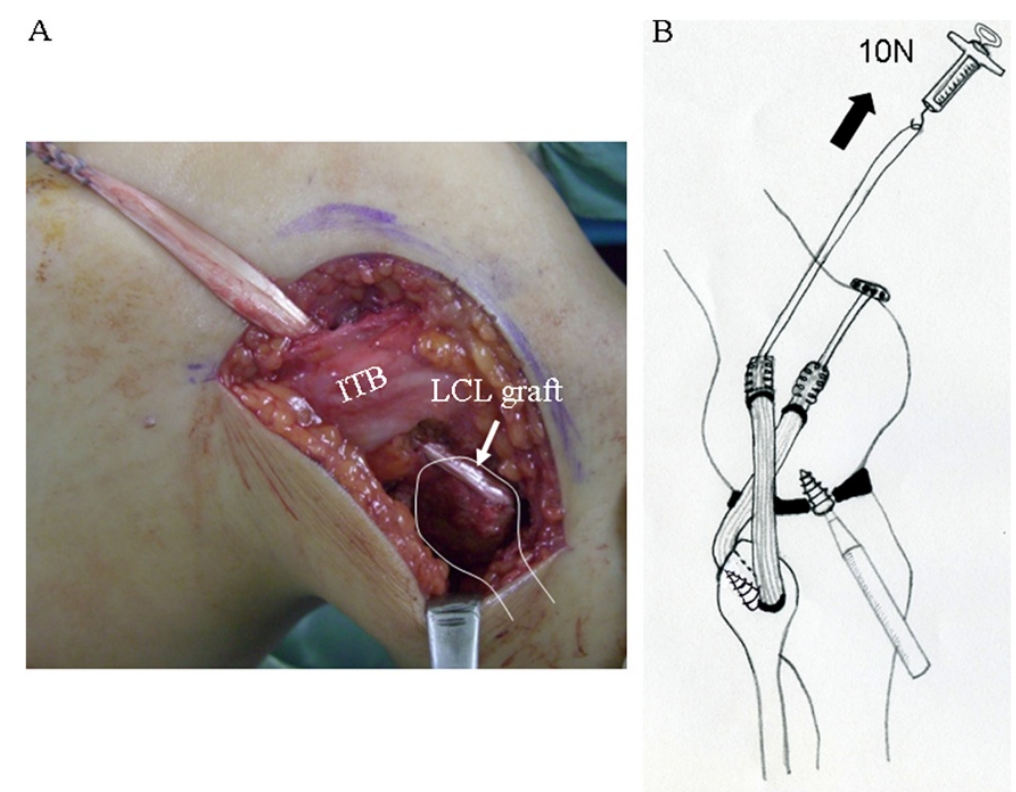

Figure 5 The graft end for the LCL is delivered under the biceps and ITB (A), and is passed into the femoral bone tunnel from the lateral epicondyle to medial cortex of the femur. The graft is then secured using an interference screw under $10 \mathrm{~N}$ force pretension with the knee in extension (B). 
A

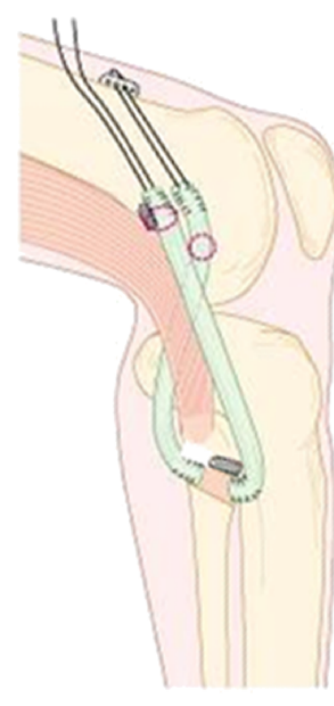

B

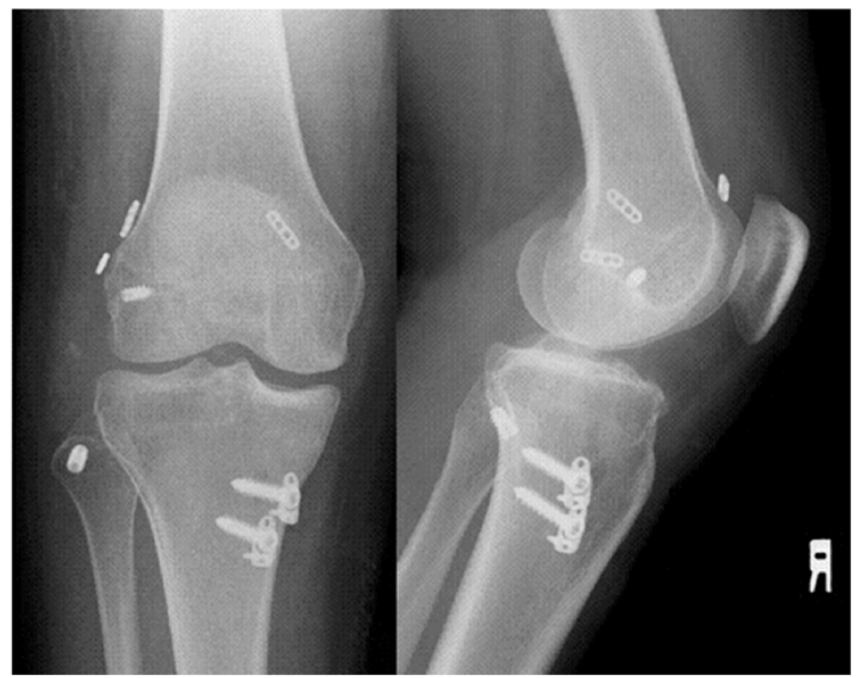

Figure 6 The reconstructed PFL and LCL cross over each other (A). Postoperative radiography shows hardware to be used for securing ACL, $L C L$, and PFL (B). When either ACL or PCL is reconstructed simultaneously, particularly with a double-bundle technique, great care should be taken with positioning of each bone tunnel to avoid overlap of these tunnels.

\section{Discussion}

Injuries to the PLC of the knee can result in severe disability due to both instability and articular cartilage degeneration. These injuries do not commonly occur in isolation, but are usually found in the setting of other injuries, such as ACL or PCL ruptures. Most authorities recommend surgical reconstruction of the PLC in combination with ACL or PCL reconstruction [11-13], since solitary reconstruction of these cruciate ligaments may results in high in situ force in the graft and concomitant PLC reconstruction potentially exerts protective effects on early failure of the cruciate ligament reconstruction.

Historically, numerous techniques for PLC reconstruction have been described, but which technique represents the best method for reconstructing physiologically functional PLC remains controversial. According to the distal insertion site of grafts for PLC, two surgical techniques are available: fibular-based techniques and combined tibial-fibular-based techniques. Larson's procedure was one of the first fibular-based techniques, and reconstructs the LCL and PFL with distal insertion sites located at the fibula [10]. Larson's procedure is still widely accepted due to the virtues of being less technically demanding and offering promising clinical results. Our technique was developed based on Larson's methods, and has been modified to reproduce a physiological tension pattern for LCL and PFL using a single ST autograft.

Tibial-fibular-based techniques have gained increasing attention due to their nature of more anatomical reconstruction capable of reconstructing all three major PLC components at each precise insertion site, but certain investigations have reported that these techniques potentiate overconstraint of posterolateral instability $[14,15]$. We believe that force distribution between the popliteus complex (PFL and popliteus tendon) and LCL is critical and should be taken into careful consideration when securing these grafts intraoperatively. A previous biomechanical study has reported that the magnitude and distribution of in situ force between the LCL and popliteus complex are affected by knee flexion angle and magnitude of posterior tibial load [16]. LCL represents a larger in situ force near full extension, decreasing with increasing flexion angle of the knee, which may explain the clinical observation that LCL is taut near full extension and relatively lax with the knee in flexion. In contrast, the popliteus complex represents a larger in situ force with the knee in flexion than with the knee in extension [12]. This force distribution pattern was employed in our modified Larson's procedure, which may thus mimic the physiological load-sharing pattern between LCL and the popliteus complex and avoid overconstraint of external and varus rotations of the tibia. Actually, LCL is secured at full extension with $10 \mathrm{~N}$, whereas the PFL is secured at $90^{\circ}$ of knee flexion with $10 \mathrm{~N}$ in our procedure. Particular emphasis in our technique is placed on intrafibular fixation of the ST graft using a small interference screw, which allows us to secure two graft limbs for LCL and PFL independently with intended tension at the intended flexion angle of 
the knee, achieving differential tension patterns for LCL and PFL.

Although favorable short-term results of tibial-fibularbased techniques have been reported [7,8], further studies documenting long-term clinical results are warranted to determine whether tibial-fibular-based techniques represent a standard optimal procedure for PLC reconstruction. At present, controversy remains as to whether all three components of the PLC should be reconstructed. Recent studies have postulated several drawbacks for tibial-fibular-based techniques, including increased technical difficulty and potential overconstraint of external and varus rotations of the knee $[14,15]$. Veltri and Warren have advocated reconstruction of PFL and LCL as sufficient to adequately control posterolateral instability such as posterior tibial translation and external and varus rotations $[17,18]$, which may support our modified Larson's method. Moreover, as the popliteus constitutively possesses a muscle belly and acts as a dynamic ligament, it is disputable that popliteus is reconstructed as a static ligament using ST tendon. Our modified Larson's method has advantages of technical simplicity and reproduction of a more physiological load-sharing pattern among grafts as compared with previously described reconstructive procedures and can offer an acceptable choice to treat chronic posterolateral instability. Further follow-up is needed to ensure that our reconstruction techniques of LCL and PFL are suitable to restore posterolateral instability of the knee.

Competing interests

The authors declare that they have no competing interests.
7. Noyes FR, Barber-Westin SD: Posterolateral knee reconstruction with an anatomical bone-patellar tendon-bone reconstruction of the fibular collateral ligament. Am J Sports Med 2007, 35:259-273.

8. Yoon $\mathrm{KH}, \mathrm{Bae} \mathrm{DK}, \mathrm{Ha} \mathrm{JH}$, Park SW: Anatomic reconstructive surgery for posterolateral instability of the knee. Arthroscopy 2006, 22:159-165.

9. LaPrade RF, Johansen S, Wentorf FA, Engebretsen L, Esterberg JL, Tso A: An analysis of an anatomical posterolateral knee reconstruction. An in vitro biomechanical study and development of a surgical technique. Arthroscopy 2004, 32:1405-1414.

10. Sidles JA, Larson RV, Garbini JL, Downey DJ, Matsen FA 3rd: Ligament length relationships in the moving knee. J Orthop Res 1988, 6:593-610.

11. Sekiya JK, Haemmerle MJ, Stabile KJ, Vogrin TM, Harner CD: Biomechanical analysis of a combined double-bundle posterior cruciate ligament and posterolateral corner reconstruction. Am J Sports Med 2005, 33:360-369.

12. Mauro CS, Sekiya JK, Stabile KJ, Haemmerle MJ, Harner CD: Double-bundle $\mathrm{PCL}$ and posterolateral corner reconstruction components are codominant. Clin Orthop Relat Res 2008, 466:2247-2254.

13. Suda $Y$, Seedhom BB, Matsumoto $H$, Otani T: Reconstructive treatment of posterolateral rotatory instability of the knee. A biomechanical study. Am J Knee Surg 2000, 13:110-116.

14. Nau T, Chevalier Y, Hagemeister N, DeGuise JA, Duval N: Comparison of 2 surgical techniques of posterolateral corner reconstruction of the knee. Am J Sports Med 2005, 33:1838-1845.

15. Markolf KL, Graves BR, Sigward SM, Jackson SR, McAllister DR: How well do anatomical reconstructions of the posterior corner restore varus stability to the posterior cruciate ligament-reconstructed knee? Am J Sports Med 2007, 35:1117-1122.

16. Hoher J, Harner CD, Vogrin TM, Baek GH, Carlin GJ, Woo SLY: In situ forces in the posterolateral structures of the knee under posterior tibial loading in the intact and posterior cruciate ligament-deficient knee. J Orthop Res 1998, 16:675-681.

17. Veltri DM, Warren RF: Operative treatment of posterolateral instability of the knee. Clin Sports Med 1994, 13(3):615-627.

18. Veltri DM, Warren RF: Posterolateral instability of the knee. J Bone Joint surg Am 1994, 76:460-472.

doi:10.1186/1758-2555-4-21

Cite this article as: Niki et al:: A modified Larson's method of posterolateral corner reconstruction of the knee reproducing the physiological tensioning pattern of the lateral collateral and popliteofibular ligaments. Sports Medicine, Arthroscopy, Rehabilitation, Therapy \& Technology 2012 4:21.

\section{Authors' contributions}

YN and HM have conceived and established the surgical techniques. YN and HE have performed the surgery. YN, HM, and TO have contributed to drafting of the manuscript. YS and YT have contributed to proof check of English. All authors have read and approved the final manuscript.

Received: 9 July 2011 Accepted: 13 June 2012

Published: 13 June 2012

\section{References}

1. Clancy WG Jr, Shelbourne KD, Zoeliner GB, Keene JS, Reider B, Rosenberg TD: Treatment of knee joint instability secondary to rupture of the posterior cruciate ligament. Report of a new procedure. J Bone Joint Surg Am 1983, 65:310-322.

2. Fanelli GC, Giannotti BF, Edson CJ: Arthroscopically assisted combined posterior cruciate ligament/posterior lateral complex reconstruction. Arthroscopy 1996, 12:521-530

3. Hughston JC, Jacobson KE: Chronic posterolateral instability of the knee. $J$ Bone Joint Surg Am 1985, 67:351-359.

4. Noyes FR, Barber-Westin SD: Surgical restoration to treat chronic deficiency of the posterolateral complex and cruciate ligaments of the knee joint. Am J Sports Med 1996, 24:415-426.

5. Albright JP, Brown AW: Management of chronic posterolateral rotatory instability of the knee: Surgical technique for the posterolateral corner sling procedure. Instr Course Lect 1998, 47:369-378.

6. Sekiya JK, Christopher AK: Posterolateral corner reconstruction of the knee: surgical technique utilizing a bifid Achilles tendon allograft and a double femoral tunnel. Arthroscopy 2005, 21:1400.e1-1400.e.5.

\section{Submit your next manuscript to BioMed Central and take full advantage of:}

- Convenient online submission

- Thorough peer review

- No space constraints or color figure charges

- Immediate publication on acceptance

- Inclusion in PubMed, CAS, Scopus and Google Scholar

- Research which is freely available for redistribution 